\title{
FILTERING OUT NOISE IN SPEECH SIGNAL WITH FIR FILTER BASED ONSINGLE COSINE TERM GENERALISED ADJUSTABLE WINDOW
}

\author{
Mbachu C. B ${ }^{1}$, Akaneme S. A. ${ }^{2}$, Muoghalu C. N. ${ }^{3}$ \\ 1, 2, 3 Department of Electrical and Electronic Engineering, Chukwuemeka Odumegwu \\ Ojukwu University,Uli, Anambra State, Nigeria. \\ 1. Email: dambac614@gmail.com 2. Email: silas.akaneme@gmail.com \\ 3. Email: cnmuoghalu@yahoo.com
}

\begin{abstract}
Speech signal is widely and effectively used globally for communication purposes in all sectors of human eandeavour. Incidentally speech signals suffer from interference by noise components when transmission through a communication channel is involved. Such noise components include Additive White Gaussian Noise (AWGN), Random Noise, power line noise, high and low frequency noise components. Without removing these noise components the speech signal quality will be compromised, implying delivery of unreliable message. In this paper a FIR filter is designed with single cosine term generalised adjustable window with to filter out high frequency noise components from speech signals. The optimal parameters of the filter are sampling frequency of $44100 \mathrm{~Hz}$ and filter order of 34 . A real voice statement, "Education is the Key to the Development of any Nation" from the lead author of this paper is captured with the in-built microphone of a laptop system, recorded in windows media audio (.wma) format and stored in one of the files of the system as speech. The optimal sampling frequency of $44100 \mathrm{~Hz}$ is applicable of the type of format of the speech signal. With "audioread" instruction the stored speech is loaded into a matlab edit window. A noise component of $4500 \mathrm{~Hz}$ and above is generated with matlab and added to the speech to imitate real corrupt speech signal. When the corrupt signal is passed through the designed filter the noise component was drastically filtered out.
\end{abstract}

Keywords: Adjustable Window, single-cosine term, high frequency noise, power spectral density.

\section{Introduction}

Some researchers have used single cosine term generalized adjustable window function to filter out noise from speech or voice signals. A generalized adjustable window is a window that assumes the appearance of some other defined and non-defined windows as the adjustment parameter is varied. In [1] Rajput and Bhadauria used a single cosine term generalised adjustable window function as shown in (1) to design low pass filter for filtering speech signals. The authors used four different values of the adjustment parameter $\boldsymbol{\alpha}$ and the performance observed. The values for adjustment used are $0.71,0.78,0.5$ (hanning window) and 0.54 (hamming window). The sampling frequency is $16000 \mathrm{~Hz}$, filter order, 33 and cutoff frequency, $3200 \mathrm{~Hz}$. The result shows that each of the resulting windows is able to significantly filter out high frequency components above $3200 \mathrm{~Hz}$, which makes them suitable for use in speech signal filtrations. There is no indication that the researcher or any other researcher used this window for filtering of speech of windows media audio (.wma) format, which is an audio signal of double column vector and recorded at a sampling frequency of $44100 \mathrm{~Hz}$. Therefore in this work, a single-cosine term generalised adjustable window will be used to design an FIR filter for filtering of speech signal of windows media audio (.wma) format.

\section{Single-Cosine Term GeneralisedAdjustable Window}

A generalised adjustable window as said above is a type of window that can take different forms depending on the value of the adjustment parameter.At certain values of the parameter it takes the appearances of known windows and at other values it represents windows of no known name or appearance. A single-cosine term generalised adjustable window has one cosine term inclusive in the function as shown in (1) [1] below in which case the adjustment parameter is $\boldsymbol{\alpha}$. When $\boldsymbol{\alpha}=\mathbf{0 . 5}$ the window becomes hanning window $[1,2,3,4]$ which is a fixed known window as in (2), and a hamming window $[5,6,78]$, another fixed known window as shown in (3) when $\boldsymbol{\alpha}=\mathbf{0 . 5 4}$. At any other value of $\boldsymbol{\alpha}$, the window takes the appearance of other windows that may or may not be known. 


$$
\begin{aligned}
& \mathrm{w}(\mathrm{n})=\alpha-(1-\alpha) \cos \left(\left(\frac{2 \pi n}{M-1}\right), 0 \leq n \leq M-1\right. \\
& \mathrm{w}(\mathrm{n})=0.5-0.5 \cos \left(\frac{2 \pi n}{M-1}\right), 0 \leq n \leq M-1 \\
& \mathrm{w}(\mathrm{n})=0.54-0.46 \cos \left(\frac{2 \pi n}{M-1}\right), 0 \leq n \leq M-1
\end{aligned}
$$

\section{Design of Low Pass FIR FilterWith Single-Cosine Term Generalised AdjustableWindow}

In this design single-cosine term generalised adjustable window function is used on FIR filter. Using the window on a FIR filter of order 34 implies that the corresponding window length is $\mathrm{M}=35$.

With specifications of filter order as 34 , cutoff frequency as $3200 \mathrm{~Hz}$ and sampling frequency as $44100 \mathrm{~Hz}$, six different values of height adjustment parameter $\boldsymbol{\alpha}=(\mathbf{0 . 0}, \mathbf{0 . 1 5}, \mathbf{0 . 5}, \mathbf{0 . 5 4}, \mathbf{0 . 6 5}$ and 0.75$)$ are considered and in each value the impulse, magnitude and phase responses of the filter are obtained and are depicted below. When $\boldsymbol{\alpha}=\mathbf{0 . 0}$ the window takes the form of a complete sine window as shown in fig. $1 \mathrm{a}$, and when $\boldsymbol{\alpha}=\mathbf{0 . 1 5}$ it becomes a different window as presented in fig. $1 \mathrm{~b}$. The sampling frequency value chosen is because the speech signal in this circumstance is recorded in windows media audio (.wma) format. The impulse, magnitude and phase responses of the filter at different values of $\boldsymbol{\alpha}$ are shown below.
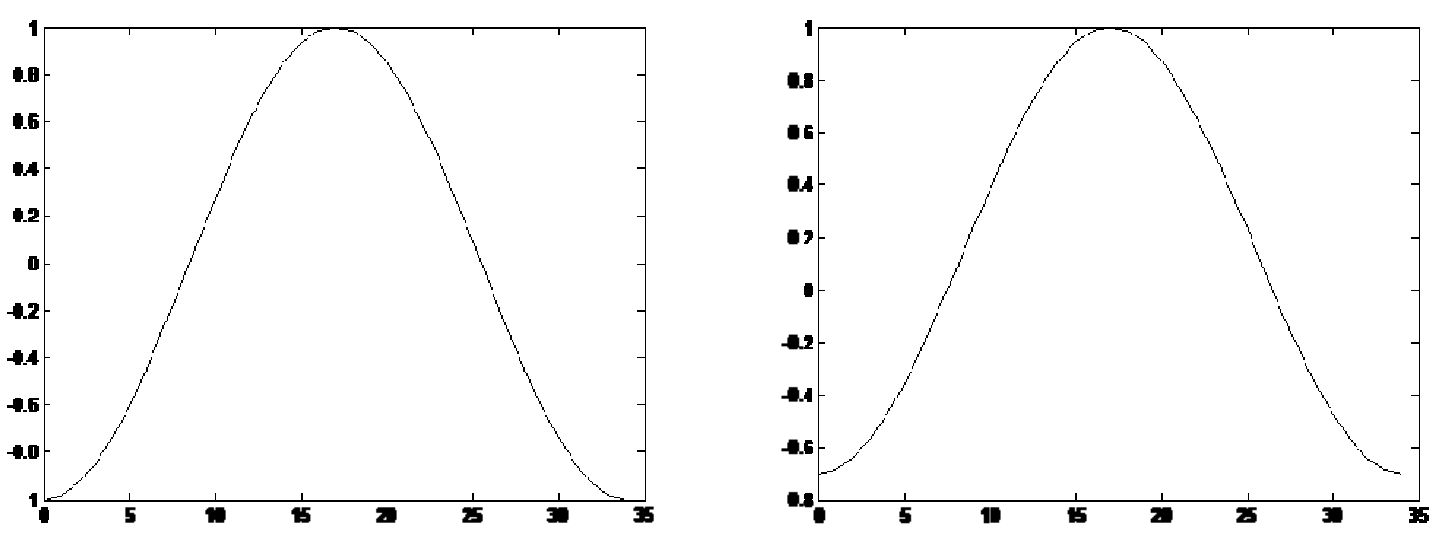

Fig. 1a: Single-cosine Term GeneralisedAdjustable Window When $\boldsymbol{\alpha}=\mathbf{0 . 0 F i g . ~ 1 b}$ : Single-cosine Term GeneralisedAdjustable Window When $\boldsymbol{\alpha}=\mathbf{0 . 1 5}$
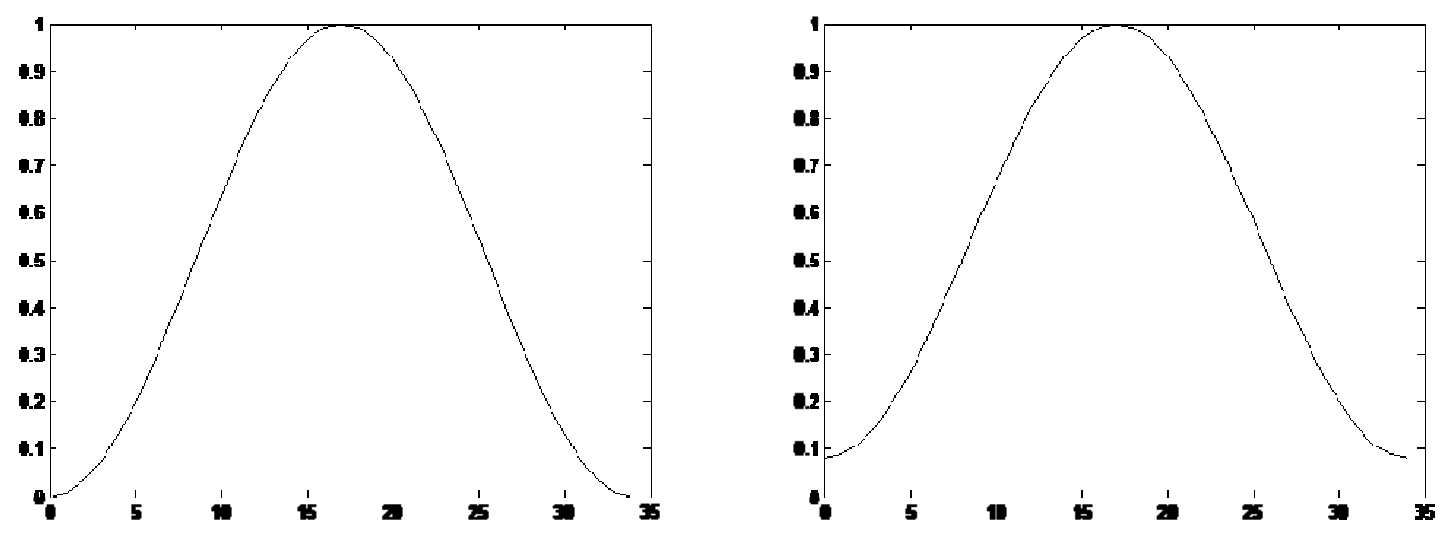

Fig. 1c: Single-cosine Term GeneralisedAdjustable Window When $\boldsymbol{\alpha}=\mathbf{0 . 5 F i g}$. 1d: Single-cosine Term GeneralisedAdjustable Window When $\alpha=0.54$ 


\subsection{Responses When $\alpha=0.0$}

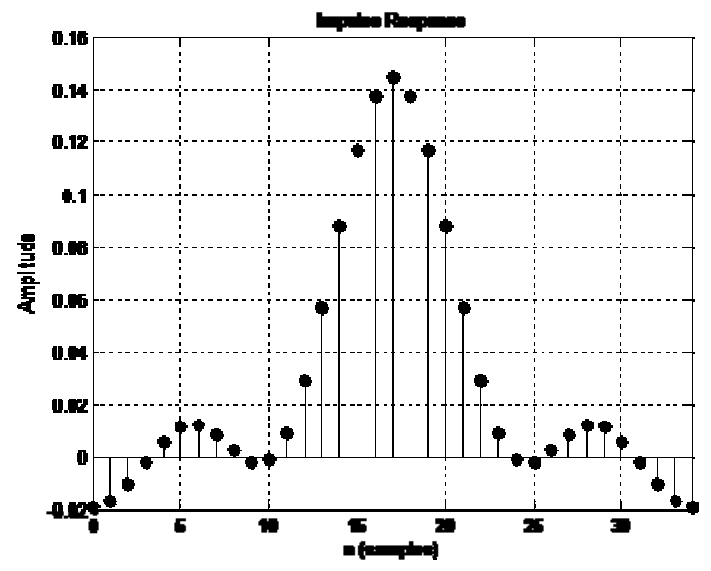

Fig 2a: Impulse Response When $\boldsymbol{\alpha}=0.0$

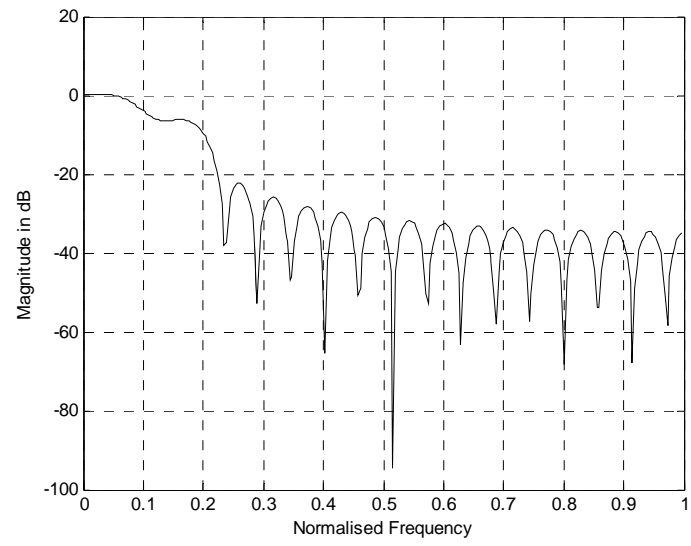

Fig 2b: Magnitude Response When $\boldsymbol{\alpha}=0.0$

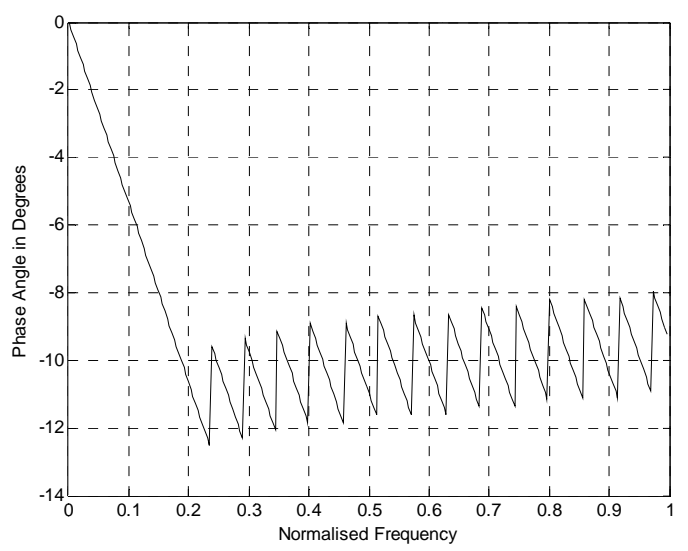

Fig 2c: Phase Response When $\boldsymbol{\alpha}=0.0$

\subsection{Responses When $\alpha=0.15$}

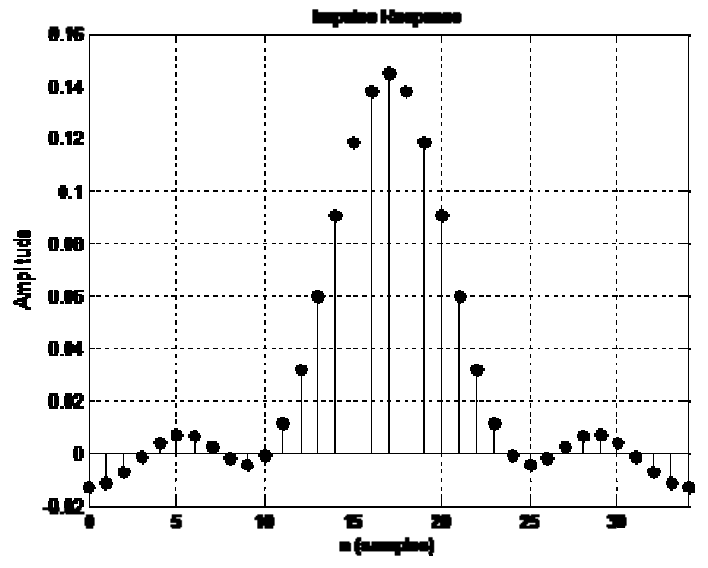

Fig 2d: Impulse Response When $\boldsymbol{\alpha}=\mathbf{0 . 1 5}$

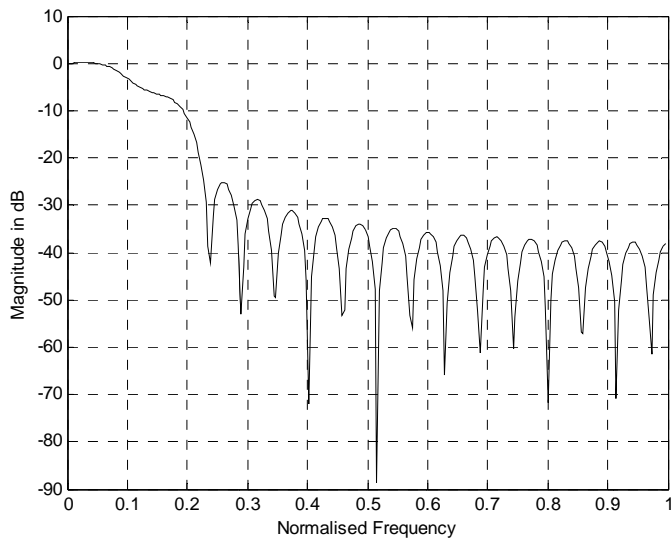

Fig 2e: Magnitude Response When $\boldsymbol{\alpha}=\mathbf{0 . 1 5}$ 


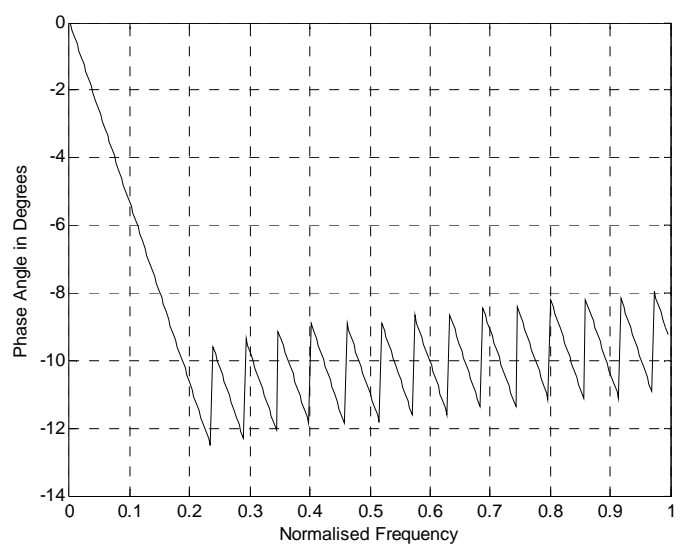

Fig 2f: Phase Response When $\boldsymbol{\alpha}=0.15$

\subsection{Responses When $\alpha=0.5$}

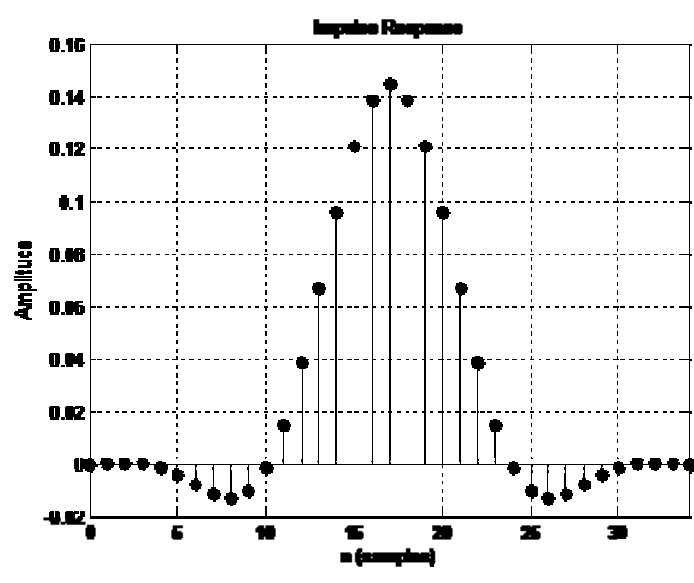

Fig 2g: Impulse Response When $\boldsymbol{\alpha}=\mathbf{0 . 5}$

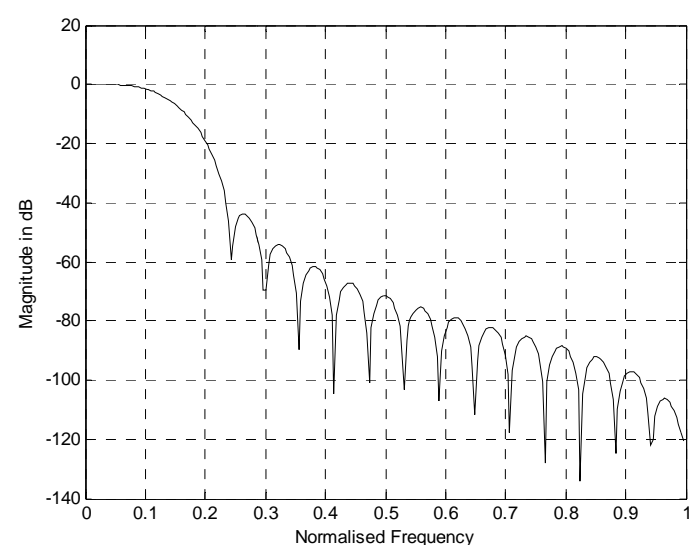

Fig 2h: Magnitude Response When $\boldsymbol{\alpha}=0.5$

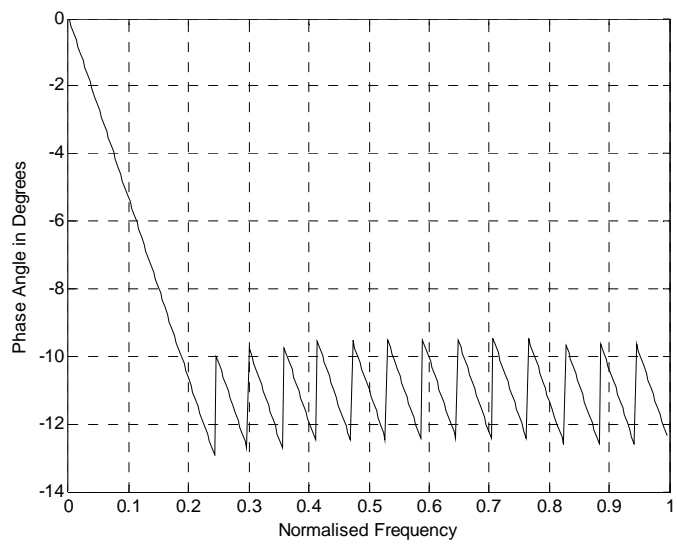

Fig 2i: Phase Response When $\boldsymbol{\alpha}=0.5$ 


\subsection{Responses When $\alpha=0.54$}

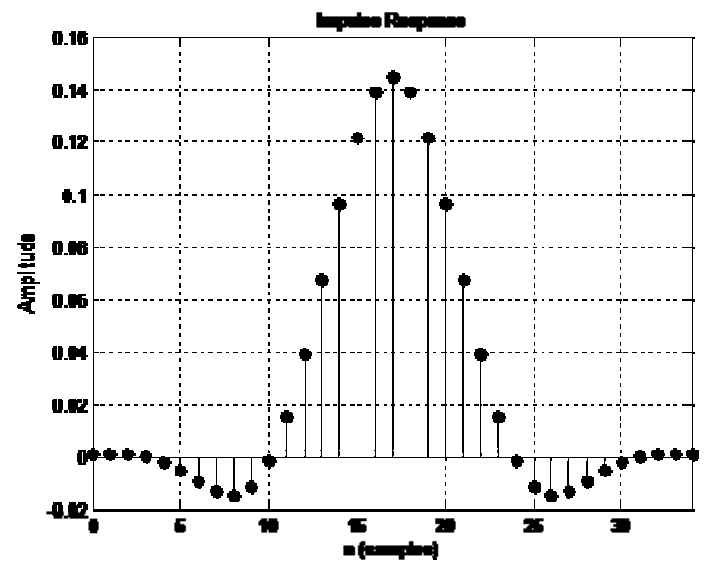

Fig 2j: Impulse Response When $\boldsymbol{\alpha}=\mathbf{0 . 5 4}$

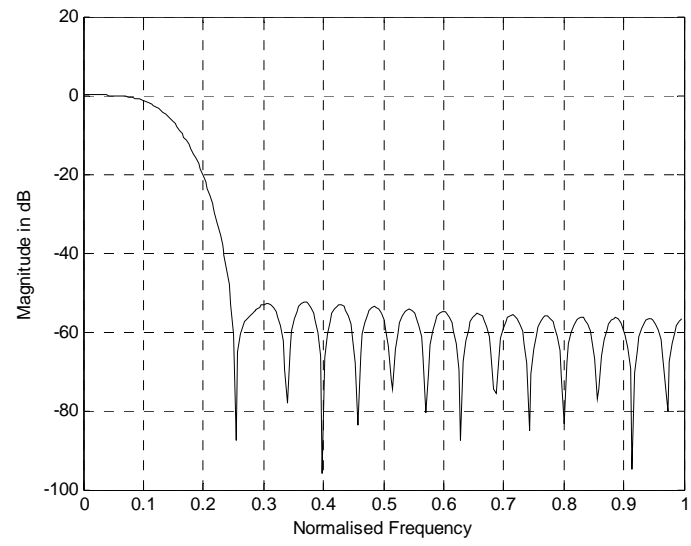

Fig 2k: Magnitude Response When $\boldsymbol{\alpha}=\mathbf{0 . 5 4}$

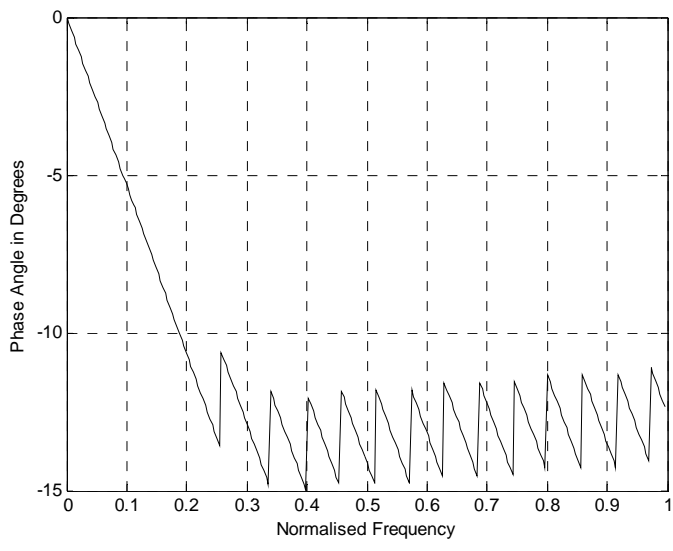

Fig 21: Phase Response When $\boldsymbol{\alpha}=\mathbf{0 . 5 4}$

\subsection{Responses When $\alpha=0.65$}

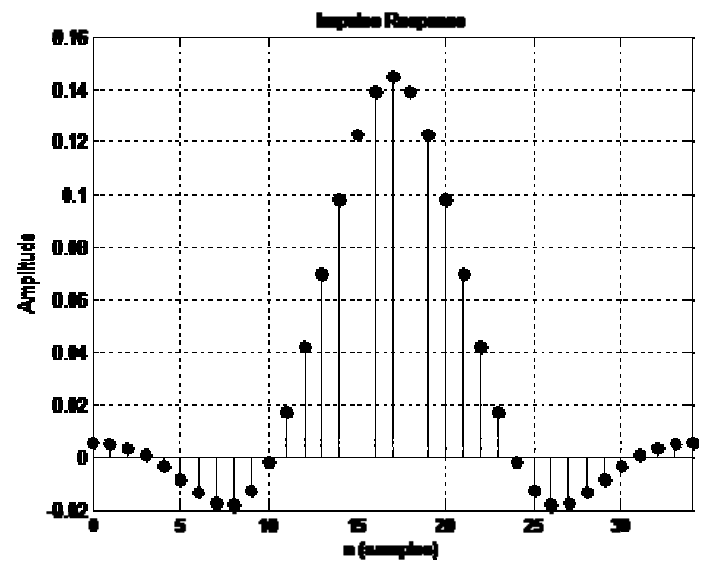

Fig 2m: Impulse Response When $\boldsymbol{\alpha}=\mathbf{0 . 6 5}$

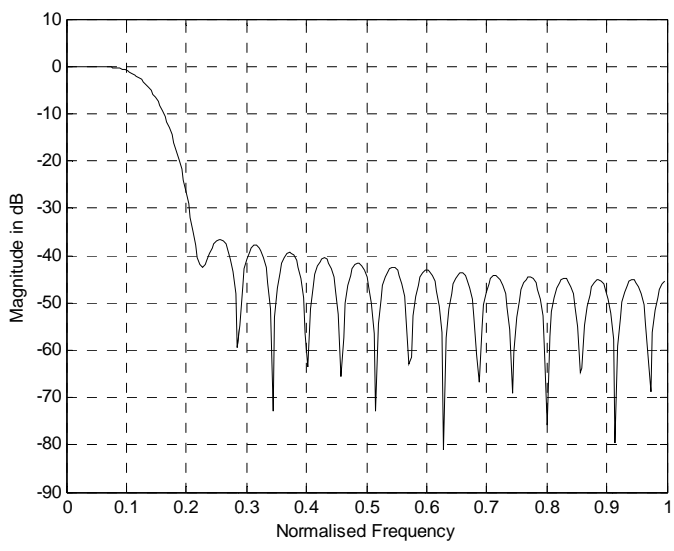

Fig 2n: Magnitude Response When $\boldsymbol{\alpha}=\mathbf{0 . 6 5}$ 


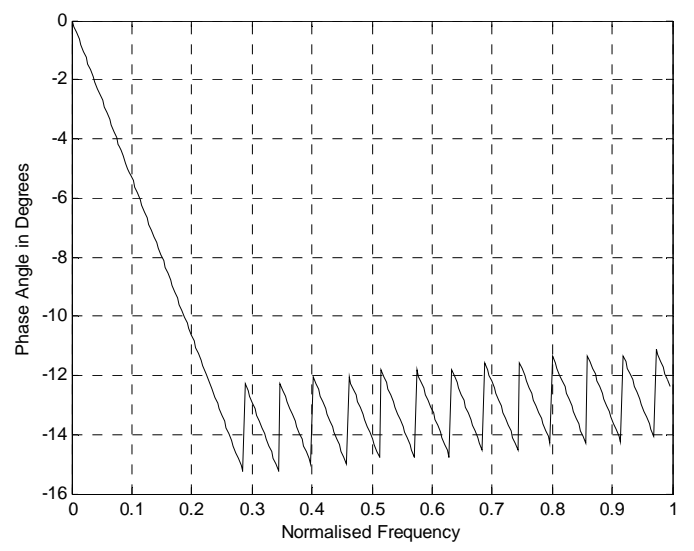

Fig 2p: Phase Response When $\mathbf{0 . 6 5}$

\subsection{Responses When $\alpha=0.75$}

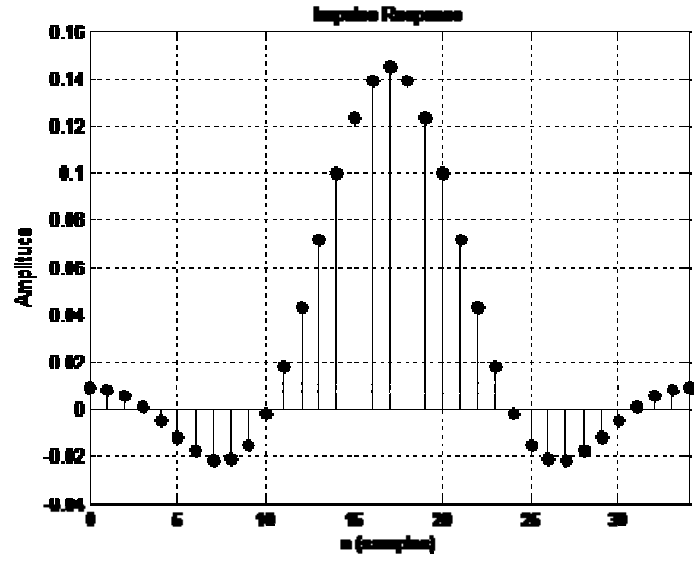

Fig 2q: Impulse Response When $\boldsymbol{\alpha}=\mathbf{0 . 7 5}$

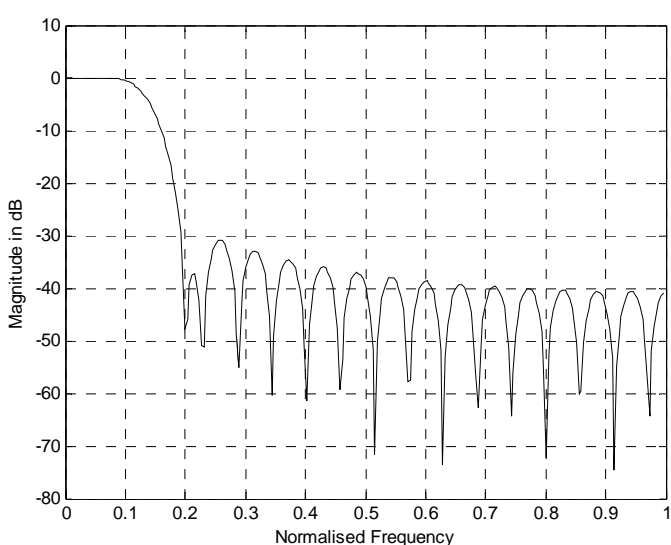

Fig 2r: Magnitude Response When $\boldsymbol{\alpha}=\mathbf{0 . 7 5}$

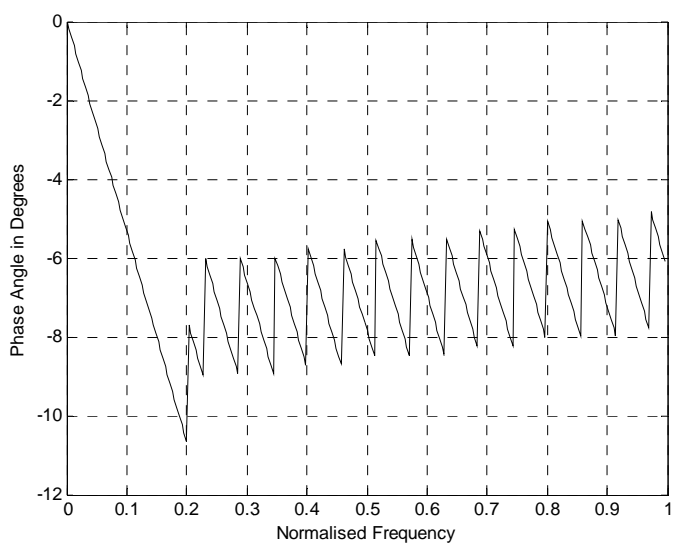

Fig 2s: Phase Response When $\boldsymbol{\alpha}=0.75$

From theresponses above, the impulse responses when $\boldsymbol{\alpha}=\mathbf{0 . 0 , 0 . 1 5 , 0 . 6 5}$ and $\mathbf{0 . 7 5}$ do not collapse completely to zeroat the last sample of the impulse response valuesas shown in figures $2 \mathrm{a}, 2 \mathrm{~d}, 2 \mathrm{~m}$ and $2 \mathrm{q}$ respectively, implying that such values are not good for the design of the desired filter. The corresponding magnitude responses of figures $2 \mathrm{~b}, 2 \mathrm{e}, 2 \mathrm{n}$ and $2 \mathrm{r}$ respectively exhibit main lobes with comparatively low magnitude values implying they will not comparatively attenuate the noise substantially. But the impulse responses when $\boldsymbol{\alpha}=\mathbf{0 . 5}$ and 0.54 exhibit stability and their main lobes have comparatively high magnitude values of $-58.15 \mathrm{~dB}$ and $62.54 \mathrm{~dB}$ respectively, implying that they will attenuate the noise substantially. The better of the two reasonable values will be determined from the power spectral densities of the filtered signals. The phase responses for all the six values of the adjustment parameterexhibit linearity within the desired bandwidth. 


\section{Results}

One of the reliable means of determining the effectiveness of filters is by analysing the power spectral density of the filtered signals.In the present circumstance a real voice statement "Education is the Key to the Development of any Nation" is transduced to electrical form and recorded in windows media audio (.wma) format and stored in a file of acomputer system as a speech signal. The signal is transferred to a matlab edit window using "audioread" instruction. In the edit window a noise component of $4500 \mathrm{~Hz}$ and above is added to the speech to constitute a corrupt speech signal. The corrupt-free speech signal is shown in fig. 3 while fig. 4 depicts the noisecomponent, and the corrupt speech signal depicted in fig.5. The corrupt speech signal is filtered with each of the designed low pass filters and the outputs recorded. Figures 6, 7, 8, 9, 10 and 11 show the speech signal after filtering. Comparing the clean speech signal of fig.3, the corrupt speech signal of fig. 5 and the filtered speech signals of fig6 to fig. 11 it can be seen that the filter reducedthe noise contained in the corrupt speech for each value of $\boldsymbol{\alpha}$. Fig. 12 to fig. 19 below are the power spectral densities of the filtered speech signal.

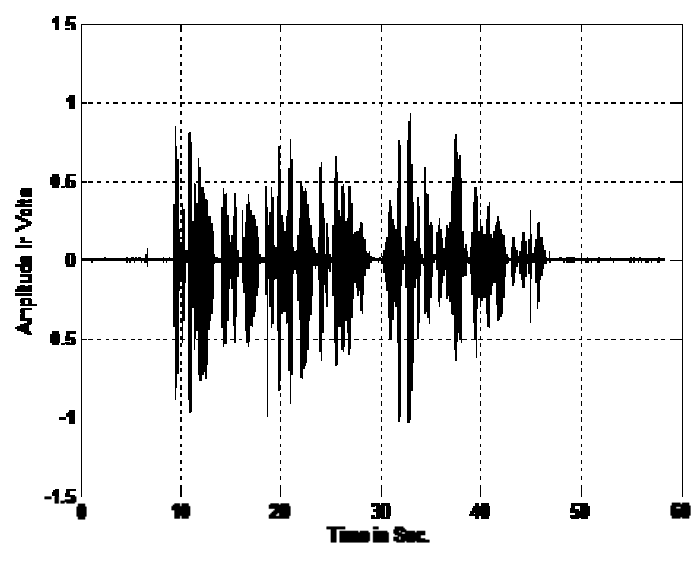

Fig. 3: Noise Free Speech Signal

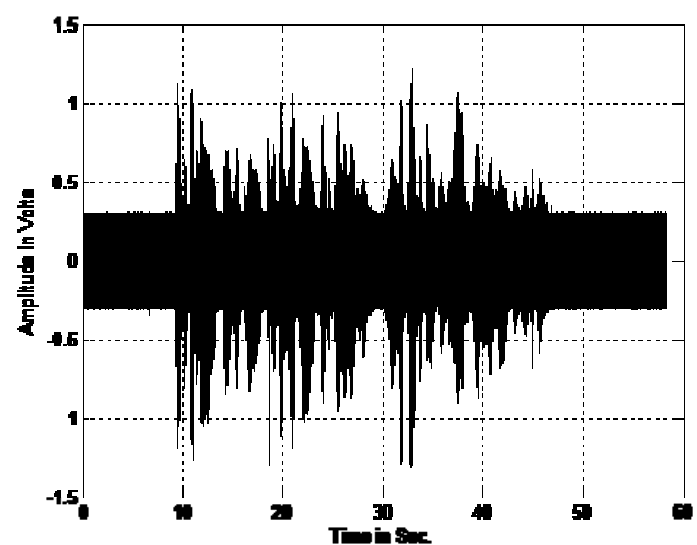

Fig. 5: Corrupt Speech signal

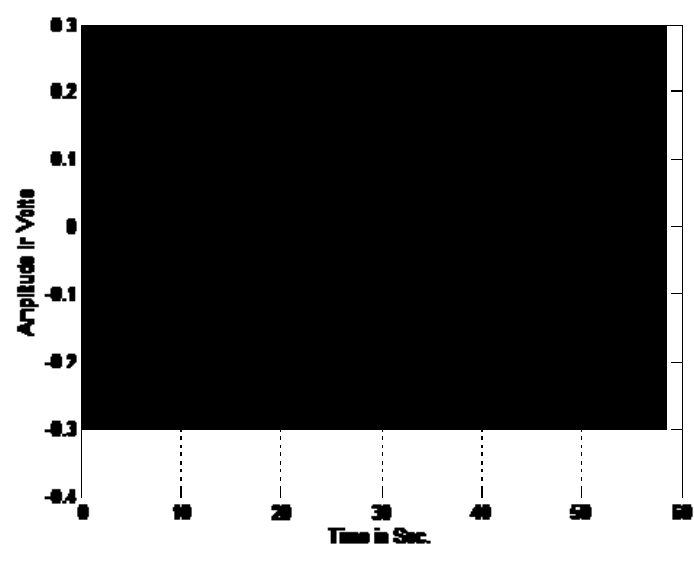

Fig. 4: Noise Signal

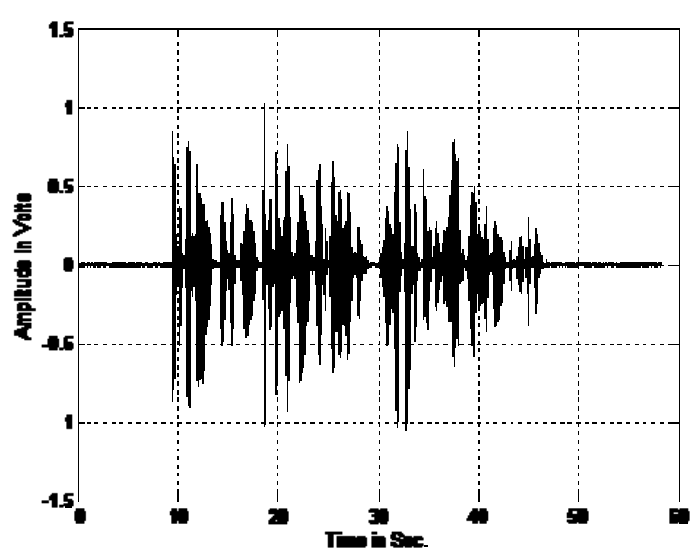

Fig.6: Speech Signal Filtered WithLow Pass Filter When $\boldsymbol{\alpha}=0.0$ 


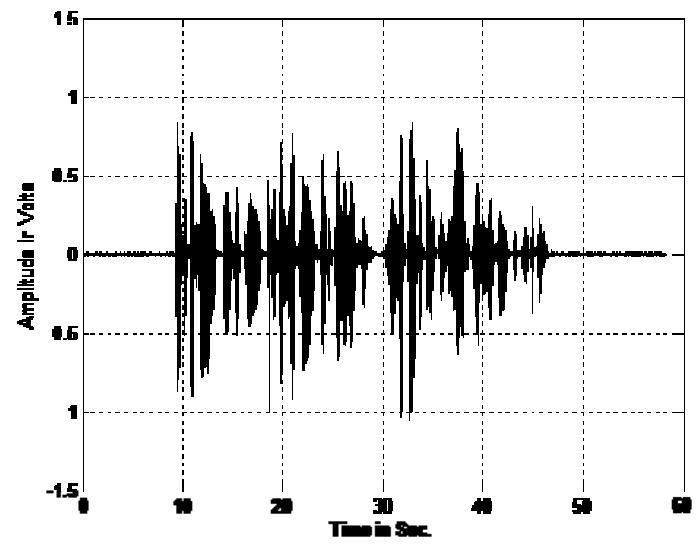

Fig.7: Speech Signal Filtered With Low Pass Filter When $\boldsymbol{\alpha}=0.15$

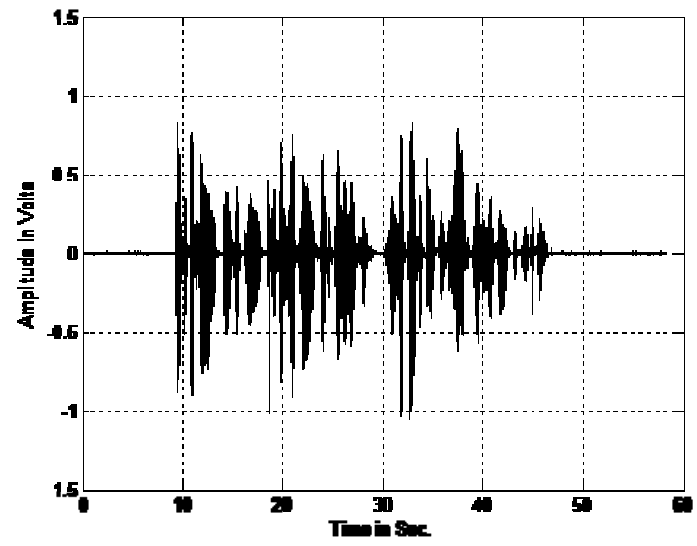

Fig.9: Speech Signal Filtered With Low Pass Filter When $\alpha=0.54$

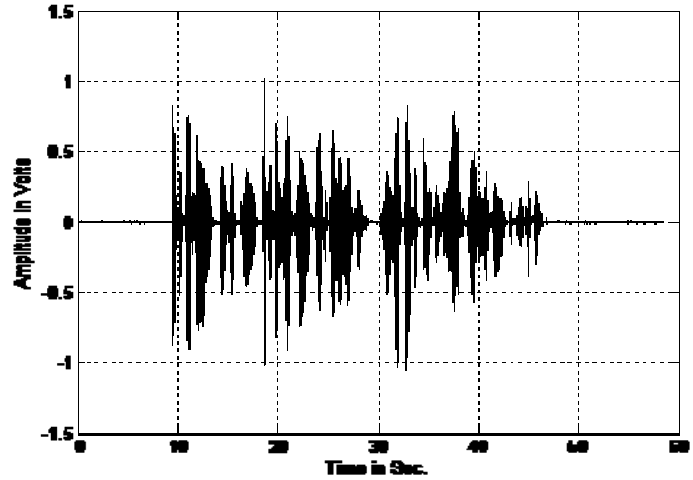

Fig.8: Speech Signal Filtered With Low Pass Filter When $\boldsymbol{\alpha}=0.5$

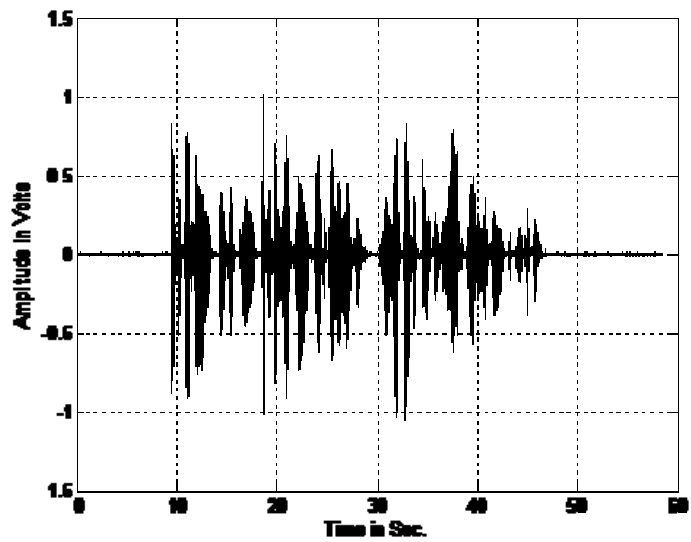

Fig.10: Speech Signal Filtered With Low Pass Filter When $\boldsymbol{\alpha}=0.65$

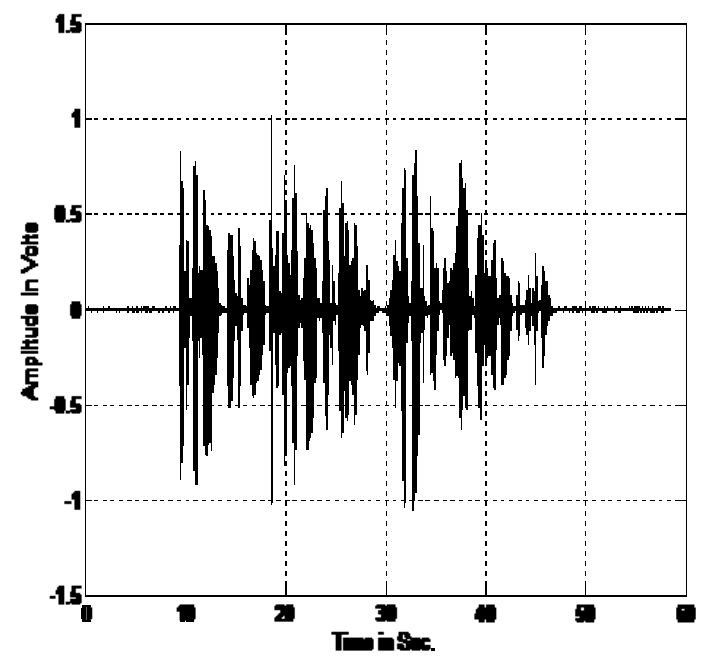

Fig.11: Speech Signal Filtered With Low Pass Filter When $\boldsymbol{\alpha}=0.75$ 


\subsection{Signal Power Level}

Power levels of the filtered signals can be determined from the power spectral densities of the signals [9, 10]. Fig. 12 is the power spectral density of a noise-free voice signal while the spectral density of the noise-corrupt voice signal is depicted in fig. 13. Fig.14 to fig.19 depicts the power spectral density of the filtered speech signals as the value of $\boldsymbol{\alpha}$ varies.From the power spectral density of the noise corrupt speech signal shown in fig. 13 the highest noise corruption occurs at a normalised frequency of 0.875 and as such we use it as a reesonable position for the analysis. Table 1 below shows the summary of the power levels in $\mathrm{dB}$ of the filtered signals as $\boldsymbol{\alpha}$ varies.

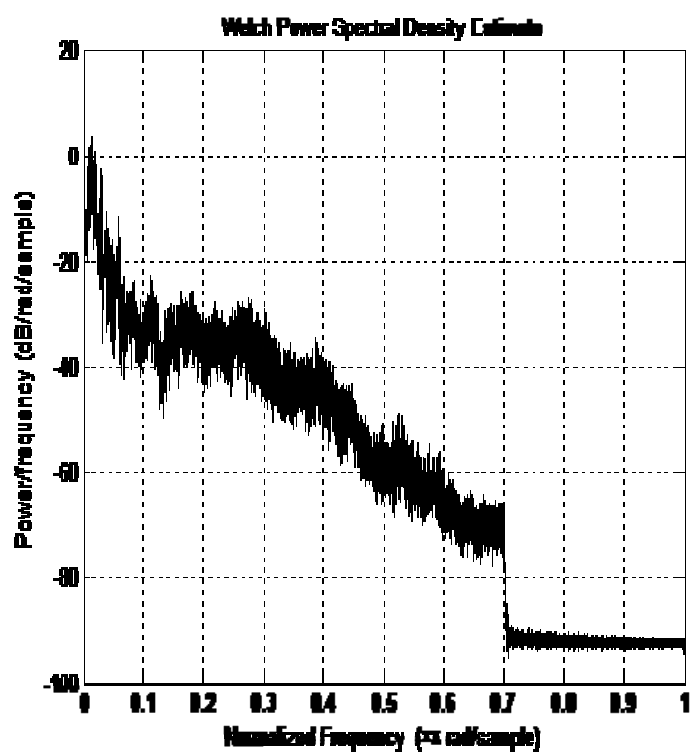

Fig.12: Power Spectral Density of Noise Free Speech Signal

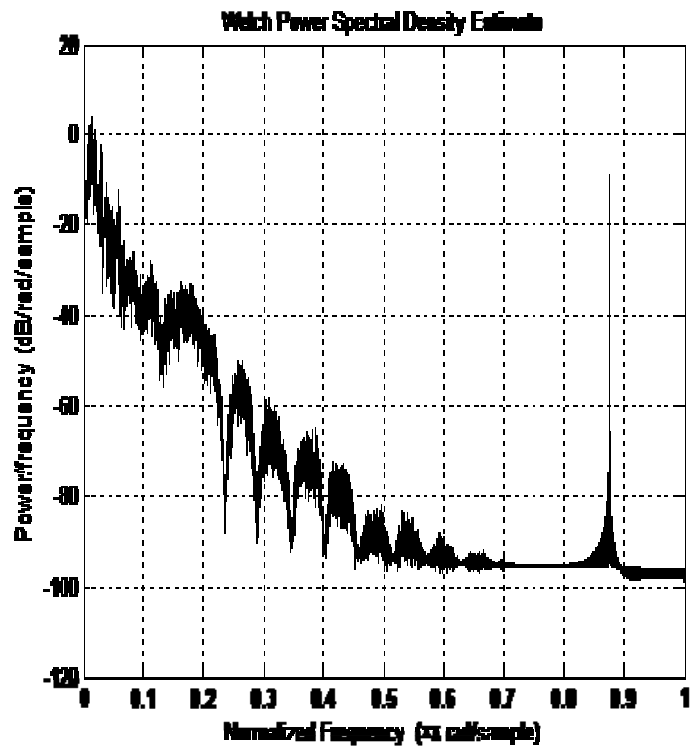

Fig.14: Power Spectral Density of Speech Signal Filtered With Low Pass Filter When $\boldsymbol{\alpha}=0.0$

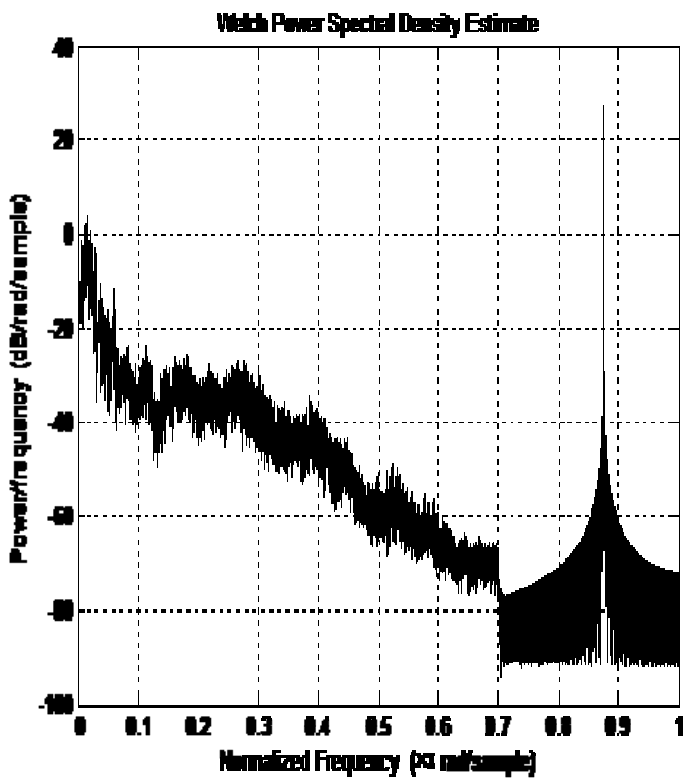

Fig.13: Power Spectral Density of Corrupt Speech Signal

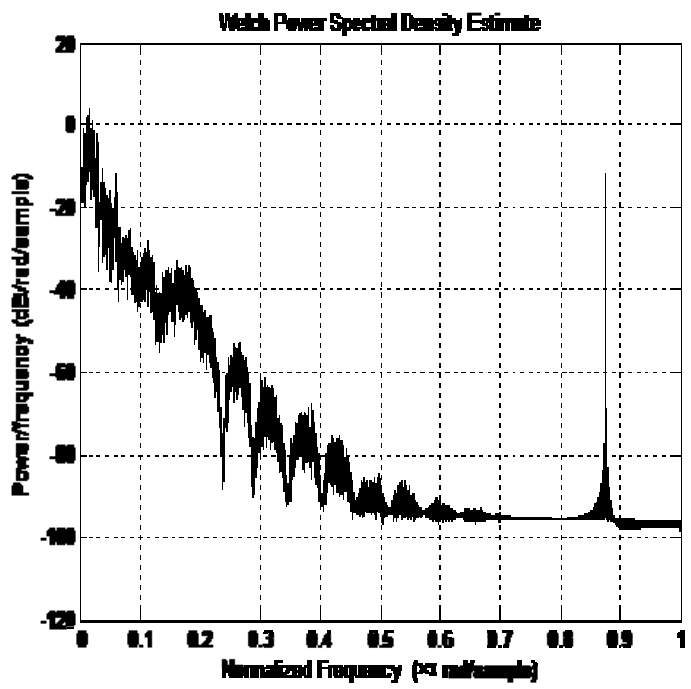

Fig.15: Power Spectral Density of Speech Signal Filtered With Low Pass Filter When $\boldsymbol{\alpha}=0.15$ 


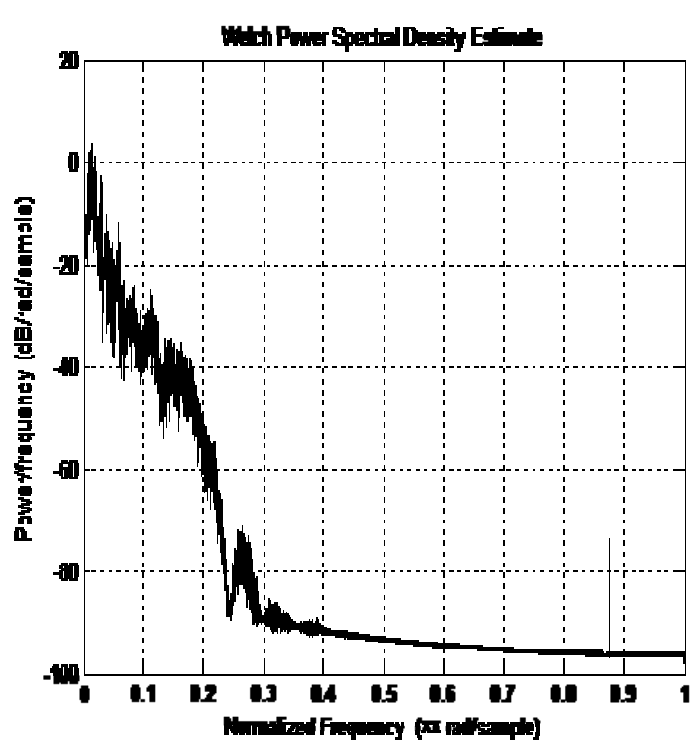

Fig.16: Power Spectral Density of Speech Signal Filtered With Low Pass Filter When $\boldsymbol{\alpha}=0.5$

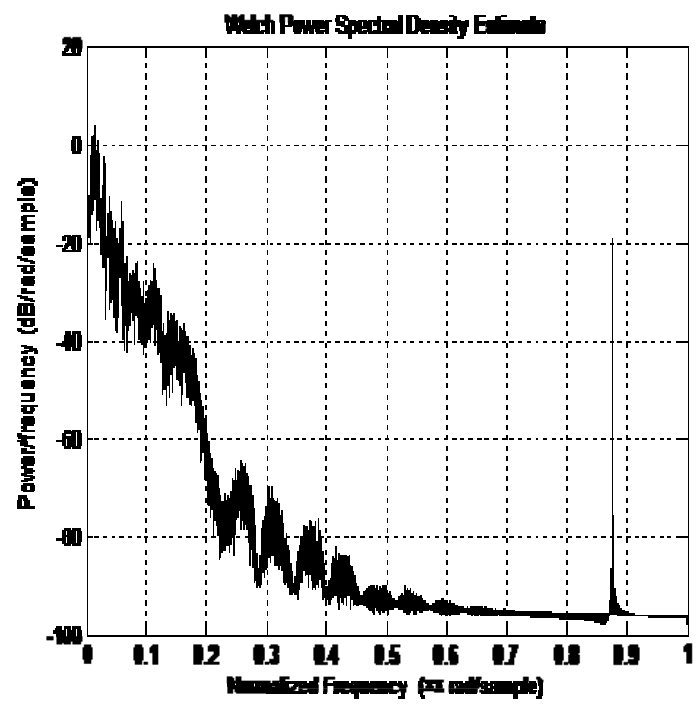

Fig.18: Power Spectral Density of Speech Signal Filtered With Low Pass Filter When $\boldsymbol{\alpha}=0.65$

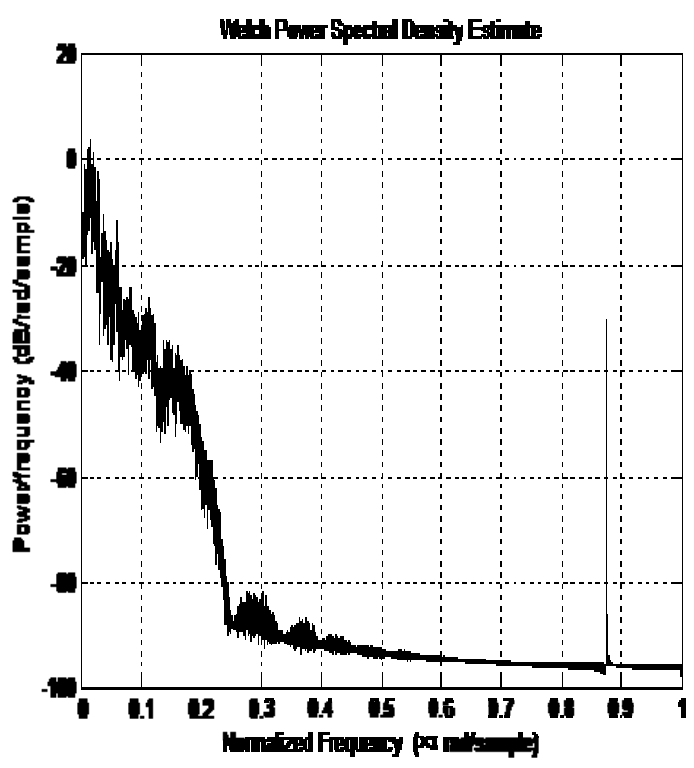

Fig.17: Power Spectral Density of Speech Signal Filtered With Low Pass Filter When $\boldsymbol{\alpha}=0.54$

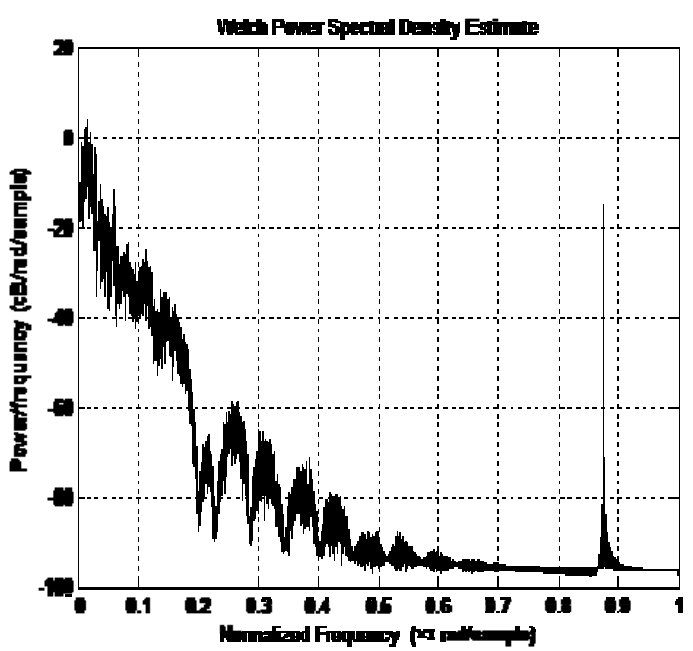

Fig.19: Power Spectral Density of Speech Signal Filtered With Low Pass Filter When $\boldsymbol{\alpha}=0.75$

Table 1: Power Levels of SpeechFiltered Signalsat 0.875 Normalised Frequency

\begin{tabular}{|c|c|}
\hline Power level of unfiltered voice & $+27.42 \mathrm{~dB}$ \\
\hline Power level of filtered voice when $\alpha=0.0$ & $-14.82 \mathrm{~dB}$ \\
\hline Power level of filtered voice when $\alpha=0.15$ & $-17.73 \mathrm{~dB}$ \\
\hline Power level of filtered voice when $\alpha=0.5$ & $-75.51 \mathrm{~dB}$ \\
\hline Power level of filtered voice when $\boldsymbol{\alpha}=54$ & $-30.92 \mathrm{~dB}$ \\
\hline Power level of filtered voice when $\boldsymbol{\alpha}=0.65$ & $-19.14 \mathrm{~dB}$ \\
\hline Power level of filtered voice when $\boldsymbol{\alpha}=7.5$ & $-16.91 \mathrm{~dB}$ \\
\hline
\end{tabular}

From table 1, power level of the corrupt speech signal is $+27.42 \mathrm{~dB}$. Comparing it with the corrupt-free speech signal power level of $-91.19 \mathrm{~dB}$ which can be determined from fig. 12 shows that noise signal added a lot of noise power of 27.42-(-91.19) $=118.61 \mathrm{~dB}$. Also from the table1 maximum attenuation of the noise is when $\boldsymbol{\alpha}=\mathbf{0 . 5}$, giving a power spectral density of $-75.51 \mathrm{~dB}$ which implies that the optimum value of the adjustment parameter is 0.5 . 


\section{Conclusion}

It can be concluded that single-cosine term generalised adjustable window is an effective window for designing FIR filters for removal of noise components from speechsignal. The adjustment parameter determines the effectiveness of the filter, and the optimum value is a function of the window length. For the speech signal, the noise type and level in this circumstance, the optimum value of the parameteris 0.5 . This value may vary if a different type of signal, noise type or window length is under consideration.

\section{References}

[1] Saurabh Singh Rajput and Dr. S. SBhaduaria. Implementation of FIR FilterUsing Adjustable Window Function and itsApplications in Speech Signal Processing. International Journal of Advances in Electrical and Electronics, Engineering, Vol. 1, No. 2, pp. 158-164.

[2] Gopika P. andSupriya Subash. Effect of High Pass Filters on Human Voice. Proceedings of $39^{\text {th }}$ IRF International Conference, $27^{\text {th }}$ March, 2016, pp. 55-59.

[3] P. Suresh Babu, Dr. D. SrinivasuluReddy, and Dr. P. V. N. Reddy . Speech Signal Analysis Using Windowing Techniques. International Journal of Emerging Trends in Engineering Research, Vol.3, No.6,2015, pp. 257-263.

[4] Rohit Patel, Er. Mukesh Kumar, Prof. A. K. Jaiswal and Er. Rohini Saxena. Design Technique of Bandpass FIR Filter Using Various Window Function. IOSR Journal of Electronics and Communication Engineering, Vol. 6, Issue 6, July- August, 2013, pp. 52-57

[5] S. Sangeetha and P. Kannan. Design and Analysis of Digital Filters for Speech Signals Using Multirate Signal Processing. ICTACT Journal on Microelectronics, Vol. 03, Issue 04, January, 2018, pp. 480-486.

[6] RiteshPawar and Rajesh Mehra. Designand performance analysis of FIR Filter forAudio Applications. International Journal ofScientific Research in Engineering and Technology, 2014, pp. 122-126.

[7] Poulami Das, Subhas Chandra, Sudip Kumar and Sankar Narayan. An Approach for Obtaining Least Noisy Signal Using Kaiser Window and and Genetic Algorithm. International Journal of Computer Applications, Vol. 150, No. 6, September, 2016, pp. 16-21.

[8] Akanksha Raj, ArshiyanazKhateeb and FakrunnisaBalaganur. Design of FIR Filter for Efficient Utilisation of Speech Signal. International Journal for Scientific Research and Development, Vol. 3, Issue 3, 2015.

[9] Mbachu C. B. and Nwosu A. W. Performance Analysis of Various Infinite Impulse Response (IIR) Digital Filters in the Reduction of Powerline Interference in ECG Signals. International Journal of Scientific and Engineering Research, Vol. 5, Issue 11, November, 2014, pp. 498-506.

[10] Marc Karam, Hasan F. Khazaal, Heshmat Aglan and Cliston Cole. Noise Removal in Speech Processing Using Spectral Subtraction. Joiurnal of Signal and Information Processing, Vol. 5, 2014, pp. 32-41. 